\title{
Differentiating Hemangioblastomas from Brain Metastases Using Diffusion-Weighted Imaging and Dynamic Susceptibility Contrast-Enhanced Perfusion-Weighted MR Imaging
}

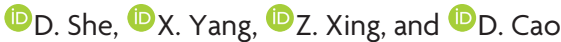

\begin{abstract}
BACKGROUND AND PURPOSE: On DWI and DSC-PWI, hemangioblastomas and brain metastases may exhibit different signal intensities depending on their cellularity and angiogenesis. The purpose of this study was to evaluate whether a hemangioblastoma can be differentiated from a single brain metastasis with DWI and DSC-PWI.
\end{abstract}

MATERIALS AND METHODS: We retrospectively reviewed DWI, DSC-PWI, and conventional MR imaging of 21 patients with hemangioblastomas and 30 patients with a single brain metastasis. Variables of minimum ADC and relative ADC were acquired by DWI and the parameter of relative maximum CBV, by DSC-PWI. Minimum ADC, relative ADC, and relative maximum CBV values were compared between hemangioblastomas and brain metastases by using the nonparametric Mann-Whitney test. The sensitivity, specificity, positive and negative predictive values, accuracy, and the area under the receiver operating characteristic curve were determined.

RESULTS: Both the minimum ADC values and relative $A D C$ ratios were significantly higher in hemangioblastomas compared with brain metastases $(P<.001$ for both minimum ADC values and relative ADC ratios). The same was true for the relative maximum CBV ratio $(P<$ .002). The threshold value of $\geq 6.59$ for relative maximum CBV provided sensitivity, specificity, and accuracy of $95.24 \%, 53.33 \%$, and $70.59 \%$, respectively, for differentiating hemangioblastomas from brain metastases. Compared with relative maximum CBV, relative ADC had high sensitivity (95.24\%), specificity (96.67\%), and accuracy (96.08\%) using the threshold value of $\geq 1.54$. The optimal threshold value for minimum $A D C$ was $\geq 1.1 \times 10^{-3} \mathrm{~mm}^{2} / \mathrm{s}$.

CONCLUSIONS: DWI and DSC-PWI are helpful in the characterization and differentiation of hemangioblastomas from brain metastases. DWI appears to be the most efficient MR imaging technique for providing a distinct differentiation of the 2 tumor types.

ABBREVIATIONS: $A D C_{\min }=$ minimum $A D C ; r A D C=$ relative $A D C ; r C B V=$ relative $C B V ; r C B V$ max $=$ relative maximum $C B V ;$ ROC = receiver operating characteristic; $A \cup C=$ area under the curve

$\mathbf{H}$ emangioblastomas are benign World Health Organization grade I tumors of vascular origin, which account for $7 \%$ of posterior fossa tumors in adults. ${ }^{1,2}$ Brain metastases are the most common type of brain malignant neoplasms, and posterior fossa metastases represent about $8.7 \%-10.9 \%$ of all brain metastases. ${ }^{3-5}$ Preoperative differentiation of hemangioblastomas and brain metastases is of high clinical relevance because surgical planning, therapeutic decisions, and prognosis vary substantially for each tumor type. In patients with hemangioblastomas, complete surgical resection is the treatment of choice, ${ }^{6}$ whereas patients with

Received December 13, 2015; accepted after revision March 18, 2016.

From the Department of Radiology, First Affiliated Hospital of Fujian Medical University, Fuzhou, P.R. China.

Please address correspondence to Dairong Cao, MD, Department of Radiology, First Affiliated Hospital of Fujian Medical University, 20 Cha-Zhong Rd, Fuzhou, Fujian 350005, P.R. China; e-mail: dairongcao@163.com

http://dx.doi.org/10.3174/ajnr.A4809 brain metastases usually undergo surgery, stereotactic surgery, whole-brain radiation therapy, chemotherapy, or combined therapy. ${ }^{7}$ Furthermore, hemangioblastomas are potentially curable and are often associated with a longer survival. ${ }^{8}$ However, brain metastases are associated with notable mortality and morbidity. ${ }^{5}$ In addition, the surgical resection of hemangioblastomas can be complicated by profuse intraoperative bleeding. Sometimes preoperative embolization of the feeding arteries may reduce the tumor blood supply, which can lessen intraoperative hemorrhage. ${ }^{9}$ In many cases, the 2 entities can be differentiated by using clinical history and conventional MR imaging. However, in some instances, particularly when the clinical findings are noncontributory and hemangioblastomas appear as solid contrast-enhancing masses with peritumoral edema, conventional MR imaging cannot be used to distinguish the 2 tumor types.

Because the clinical management and prognosis of these 2 types of tumor are vastly different, it is important to distinguish them with certainty. Advanced MR imaging approaches includ- 
ing DWI and DSC-PWI might complement physiologic information in addition to that obtained with conventional MR imaging. DWI could assess the Brownian movement of water in the microscopic tissue environment and reflect cellularity of the tissue by ADC values, which may aid conventional MR imaging in the characterization of brain tumors and other intracranial diseases. ${ }^{10-12}$ DSC-PWI that provides noninvasive morphologic and functional information of the tumor microvasculature can be useful in the preoperative diagnosis and grading of brain tumors. MR imaging parameters of relative cerebral blood volume (rCBV) have become some of the most robust hemodynamic variables used in the characterization of the brain tumors. ${ }^{13-15}$ Hemangioblastomas may present with histopathologic structures vastly different from those found in brain metastases. Thus, the application of DWI and DSC-PWI may better evaluate and discriminate the cytostructural and hemodynamic differences between hemangioblastomas and brain metastases.

Only a few small studies have evaluated the advanced MR imaging features of a hemangioblastoma, ${ }^{12,16,17}$ particularly when assessing their differentiation from a single brain metastasis. ${ }^{18}$ The purpose of this study was to evaluate whether a hemangioblastoma can be differentiated from a single brain metastasis with DWI and DSC-PWI.

\section{MATERIALS AND METHODS}

\section{Patients}

The institutional review board of our hospital approved this retrospective study, and the requirement for patient informed consent was waived due to its retrospective nature. Potentially eligible patients with histologically confirmed hemangioblastomas and brain metastases from January 2010 through September 2015 were identified. For the selection of appropriate patients, those with multiple brain lesions, hemorrhagic lesions, and previously treated or nonenhancing tumor were excluded. Pretreament MR images of consecutive patients were reviewed retrospectively, and DWI and DSC-PWI were requested in addition to conventional MR imaging.

\section{MR Imaging Techniques}

MR imaging examinations were performed in the routine clinical work-up on a 3T MR imaging system (Magnetom Verio Tim; Siemens, Erlangen, Germany) by using an 8-channel head matrix coil. The conventional MR imaging protocols consisted of the following sequences: axial T2-weighted turbo spin-echo imaging (TR/TE, 4000 /96 ms), axial T1-weighted spin-echo imaging (TR/ TE, $250 / 2.48 \mathrm{~ms}$ ), axial fluid-attenuated inversion recovery (TR, $9000 \mathrm{~ms}$; TE, $94 \mathrm{~ms}$; TI, $2500 \mathrm{~ms}$ ), and contrast-enhanced gradient-echo T1-weighted imaging (TR/TE, $250 \mathrm{~ms} / 2.48 \mathrm{~ms}$ ) in 3 orthogonal planes, which was acquired following the acquisition of the DSC-PWI sequences. FOV at $220 \times 220 \mathrm{~mm}$, section thickness of $5 \mathrm{~mm}$, and intersection gap of 1 were uniform in all sequences. Before the injection of contrast material, DWI was performed in the axial plane with a spin-echo echo-planar sequence. The imaging parameters used were as follows: TR/TE, 8200/102 ms; NEX, 2.0; FOV, $220 \times 220$; section thickness, $5 \mathrm{~mm}$; intersection gap, $1 \mathrm{~mm}$. The b-values were 0 and $1000 \mathrm{~s} / \mathrm{mm}^{2}$ with diffusion gradients encoded in the 3 orthogonal directions to generate
3 sets of diffusion-weighted images ( $\mathrm{x}^{-}, \mathrm{y}^{-}$, and $\mathrm{z}$-directions). Processing of the ADC map was generated automatically on the MR imaging scanner.

The DSC-PWI was obtained with a gradient-recalled $\mathrm{T}^{*}$ weighted echo-planar imaging sequence. The imaging parameters used were as follows: TR/TE, 1000-1250/54 ms; flip angle, 35; FOV, $220 \times 220$; NEX, 1.0; section thickness, $5 \mathrm{~mm}$; intersection gap, $1 \mathrm{~mm}$. During the first 3 phases, images were scanned before injecting the contrast material to establish a precontrast baseline. When the scan was to the fourth phase of DSC-PWI, $0.1 \mathrm{mmol} / \mathrm{kg}$ body weight of gadopentetate dimeglumine was injected intravenously with an MR imaging-compatible power injector at a flow rate of $5 \mathrm{~mL} / \mathrm{s}$ through an intravenous catheter placed in the right or left antecubital vein, followed by $20 \mathrm{~mL}$ of continuous saline flush. The series of 20 sections, 60 phases, and 1200 images was obtained in 1 minute 36 seconds.

\section{Data Processing}

All imaging assessments were performed on an off-line Siemens syngo MR B19 work station (NUMARIS/4) with standard software. For evaluation of conventional MR images, a neuroradiologist who was blinded to the tumor histology retrospectively reviewed the images and evaluated each lesion on the basis of location, tumor characteristics (solid-cystic or solid), presence or absence of signal void on T2-weighted imaging, and contrast enhancement pattern (homogeneous or heterogeneous). For evaluation of DWI data, qualitative assessment of the signal intensity in the enhancing solid portion of the tumors on contrast-enhanced T1-weighted images was performed. The signal intensity of the tumor was classified as hypointense, isointense, or hyperintense compared with normal white matter. The ADC values were measured by manually placing ROIs inside the tumor regions on the ADC maps. At least 5 small round ROIs $\left(30-40 \mathrm{~mm}^{2}\right)$ were placed inside the tumors on the ADC maps, and the minimum $\mathrm{ADC}$ values $\left(\mathrm{ADC}_{\mathrm{min}}\right)$ were taken into consideration. The ROI placements were made from the enhancing solid portion of the lesion, avoiding hemorrhagic, necrotic, cystic, or apparent blood vessel regions that might influence the ADC values. For each patient, the enhancing solid portion of the tumor was identified on contrast-enhanced axial T1-weighted images and matching ADC maps. The same method was applied to a corresponding area in the contralateral unaffected white matter judged as normal on both T2- and contrast-enhanced T1-weighted images. The relative $\mathrm{ADC}$ ( $\mathrm{rADC}$ ) ratios of the tumors were calculated as the ratios of the minimum ADC of the tumors divided by the mean ADC of the contralateral unaffected white matter. $\mathrm{ADC}_{\text {min }}$ values were expressed as $\times 10^{-3} \mathrm{~mm}^{2} / \mathrm{s}$.

For evaluation of DSC-PWI data, whole-brain CBV maps were generated by using a single-compartment model and an automated arterial input function. Measurements of $\mathrm{rCBV}$ values were performed with the same ROIs as those used for ADC measurements, and the maximum $\mathrm{rCBV}\left(\mathrm{rCBV}_{\max }\right)$ values were taken into consideration. To minimize variances in the $\mathrm{CBBV}$ values in an individual patient, we calculated the rCBV ratios of the tumors as the ratios of the rCBV values from ROIs of the tumors divided by the mean $\mathrm{rCBV}$ value of the contralateral unaffected white matter. The ROIs for the ADC and rCBV measurements were not 
identical and were not from the same solid contrast-enhancing region of the tumor in each single patient. The signal intensity on DWI, $\mathrm{ADC}_{\text {min }}$, rADC, and $\mathrm{rCBV}_{\text {max }}$ parameters was acquired by another neuroradiologist who was experienced with diffusion and perfusion data acquisition and blinded to the tumor histology. This method for the measurements of maximal abnormality has been shown to provide the highest interobserver and intraobserver reproducibility. ${ }^{19}$

\section{Data Analysis}

All hemangioblastoma and brain metastasis parameters are presented as means $\pm \mathrm{SDs}$. Comparisons of $\mathrm{ADC}_{\min }\left(\times 10^{-3} \mathrm{~mm}^{2} /\right.$ $\mathrm{s}), \mathrm{rADC}$, and $\mathrm{rCBV}_{\max }$ values between patients with hemangioblastomas and those with brain metastases were made with nonparametric Mann-Whitney statistical tests. Comparisons of the signal intensity on DWI between patients with hemangioblastomas and those with brain metastases were made with $\chi^{2}$ tests. The receiver operating characteristic (ROC) analysis curves were obtained to decide the diagnostic accuracy and optimum cutoff value of $\mathrm{ADC}_{\text {min }}, \mathrm{rADC}$, and $\mathrm{rCBV}_{\text {max }}$ for differentiating hemangioblastomas from brain metastases. The sensitivity, specificity, positive predictive value, negative predictive value, accuracy, and area under the curve (AUC) based on optimum thresholds for $\mathrm{ADC}_{\text {min }}$ and $\mathrm{rCBV}_{\max }$ were calculated to differentiate hemangioblastomas from brain metastases. The cutoff values chosen were those that provided optimal sensitivity and specificity jointly. In addition, comparison of AUCs for different quantitative variables was made with a $Z$-test. Statistical analysis was performed in Excel 2007 (Microsoft, Redmond, Washington) and the Statistical Package for the Social Sciences (Version 17.0; IBM, Armonk, New York). $P$ values $<.05$ were statistically significant.

Table 1: The main clinical features of hemangioblastomas and brain metastases

\begin{tabular}{lcc}
\hline & Hemangioblastomas & $\begin{array}{c}\text { Brain } \\
\text { Metastases }\end{array}$ \\
\hline No. of patients (male/female) & $10: 11$ & $19: 11$ \\
Mean age (yr) & $41.1 \pm 15.8$ & $57.5 \pm 12.0$ \\
Localization & 19 & 5 \\
Cerebellar hemisphere & 1 & 12 \\
Frontal lobe & 1 & \\
Choroid fissure & & 8 \\
Parietal lobe & 1 \\
Occipital lobe & 4 \\
Temporal lobe & \\
Origin of brain metastases & 19 \\
Lung carcinoma & 2 \\
Breast carcinoma & 2 \\
Gastric carcinoma & 1 \\
Esophagus carcinoma & 2 \\
Liver carcinoma & 1 \\
Melanoma & 1 \\
Colon carcinoma & 2 \\
Carcinoma of unknown origin & & \\
\hline
\end{tabular}

\section{RESULTS}

Fifty-one histologically proved cases, including 21 cases with hemangioblastomas and 30 cases with brain metastases, were enrolled in this study. The main clinical features of the hemangioblastomas and brain metastases are summarized in Table 1. The characteristics of hemangioblastomas and brain metastases on conventional MR imaging are shown in Table 2.

The $\mathrm{ADC}_{\text {min }}$ values, $\mathrm{rADC}$ ratios, and $\mathrm{rCBV}_{\text {max }}$ calculated for hemangioblastomas and brain metastases are given in Table 3. On DWI, the signal intensity in the solid portions of hemangioblastomas were hypointense $(n=3)$, isointense $(n=13)$, and hyperintense $(n=5)$ relative to normal-appearing white matter. Conversely, the signal intensity of brain metastases was hypointense $(n=1)$, isointense $(n=3)$, and hyperintense $(n=26)$. The signal intensity in the solid contrast-enhancing portions of hemangioblastomas was significantly lower than that of brain metastases $(P<.001)$. Both the $\mathrm{ADC}_{\text {min }}$ values and $\mathrm{rADC}$ ratios were significantly higher in hemangioblastomas compared with brain metastases (Table 3 and Figs $1 B$ and $2 B$ ).

The $\mathrm{rCBV}_{\max }$ values in patients with hemangioblastomas were significantly higher than those in patients with brain metastases (Table 3 and Figs $1 C$ and 2C). Twenty of 21 (95.23\%) hemangioblastomas showed markedly elevated perfusion ( $\mathrm{rCBVmax}>$ 6.0 ), while $16 / 30(53.33 \%$ ) brain metastases showed significantly elevated perfusion.

The results of the ROC curve analysis are shown in Table 4 and Fig 3, which summarize the sensitivity, specificity, positive predictive values, negative predictive values, accuracy, and AUC for the different quantitative parameters for differentiating hemangioblastomas from brain metastases. From the ROC analysis, the highest AUC was obtained for rADC compared with rCBVmax ( 0.971 versus $0.756, Z=3.075, P=.002$ ) in the differentiation of hemangioblastomas and brain metastases, which corresponded to histopathologic findings in $95.24 \%$ (20 of 21) of patients with hemangioblastomas and $96.67 \%$ (29 of 31) of those with brain metastases. With a threshold value of $\geq 1.54$ for $\mathrm{rADC}$ values, the accuracy in the diagnosis of hemangioblastomas was $96.08 \%$.

\section{DISCUSSION}

In our study, we used 2 diagnostic parameters derived from DWI and DSC-PWI to differentiate hemangioblastomas and brain metastases, which are sometimes not distinguishable with conventional MR imaging. Our study showed that patients with heman-

Table 3: Comparison of the hemangioblastomas and brain metastases with regard to the variables of interest (mean \pm SD)

\begin{tabular}{lccc} 
& Hemangioblastomas & Brain Metastases & $\begin{array}{c}P \\
\text { Value }\end{array}$ \\
\hline $\mathrm{ADC}_{\min }$ & $1.5 \pm 0.52 \times 10^{-3} \mathrm{~mm}^{2} / \mathrm{s}$ & $0.79 \pm 0.21 \times 10^{-3} \mathrm{~mm}^{2} / \mathrm{s}$ & $<.001$ \\
$\mathrm{rADC}$ & $2.3 \pm 0.76$ & $1.12 \pm 0.32$ & $<.001$ \\
$\mathrm{rCBV}_{\max }$ & $8.5 \pm 2.45$ & $6.4 \pm 2.0$ & .002 \\
\hline
\end{tabular}

Table 2: Characteristics of hemangioblastomas and brain metastases on conventional MR imaging

\begin{tabular}{llcccr} 
& & & & \multicolumn{2}{c}{ Contrast-Enhancement Pattern } \\
\cline { 3 - 6 } \multicolumn{1}{c}{ Tumor } & Solid-Cystic & Solid & Presence/Absence of SV & Homogeneous & Heterogeneous \\
\hline Hemangioblastomas & $12 / 21(57.1 \%)$ & $9 / 21(42.9 \%)$ & $12 / 9$ & $15 / 21(71.5 \%)$ & $6 / 21(28.5 \%)$ \\
Brain metastases & $6 / 30(20 \%)$ & $24 / 30(80 \%)$ & $6 / 24$ & $5 / 30(16.7 \%)$ & $25 / 30(83.3 \%)$ \\
\hline
\end{tabular}

Note:-SV indicates signal void.

1846 She Oct 2016 www.ajnr.org 


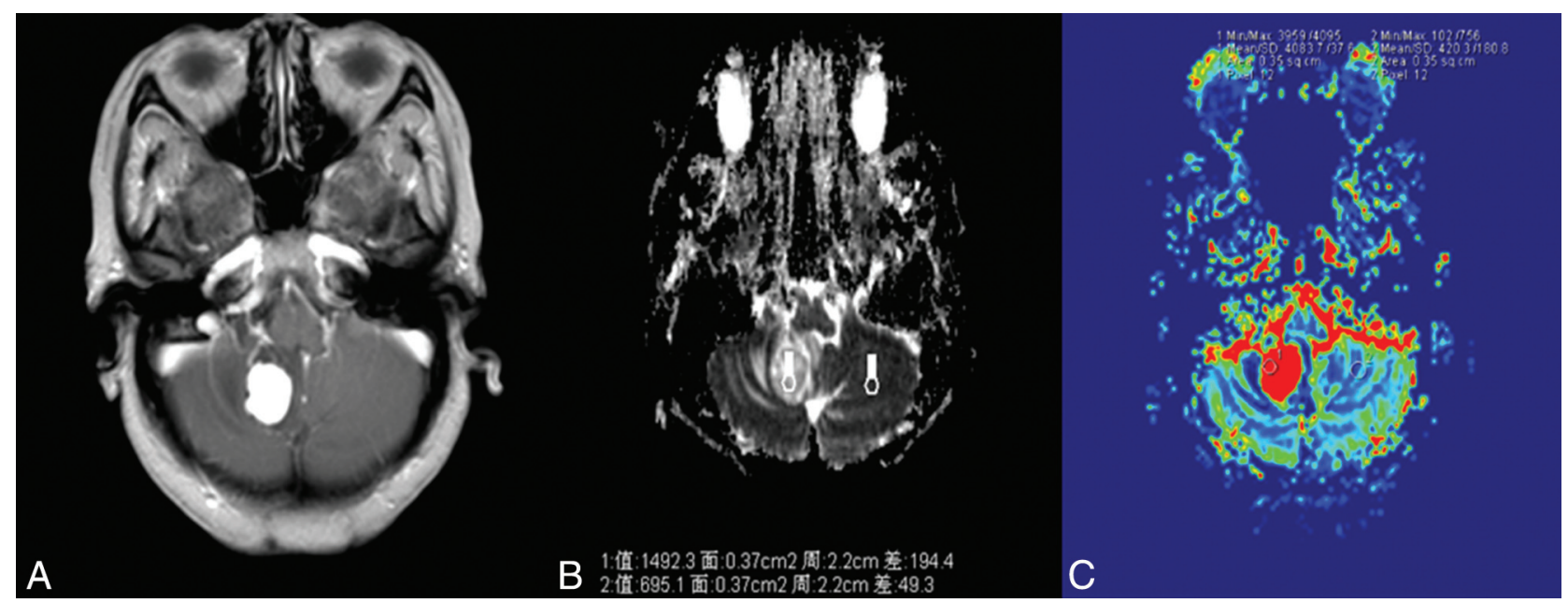

FIG 1. A 54-year-old woman with hemangioblastoma. A, Contrast-enhanced axial T1-weighted image demonstrates an obvious contrastenhancing lesion on the right cerebellar hemisphere. $B, A$ corresponding $A D C$ map shows the tumor with an increased $A D C$ value $(A D C$ min $=$ $\left.1492.3 \times 10^{-3} \mathrm{~mm}^{2} / \mathrm{s}, \mathrm{rADC}=2.15\right)$. C, Correlative color CBV image shows significant elevated perfusion with the calculated rCBV $\mathrm{max}_{\text {of }} 9.72$.

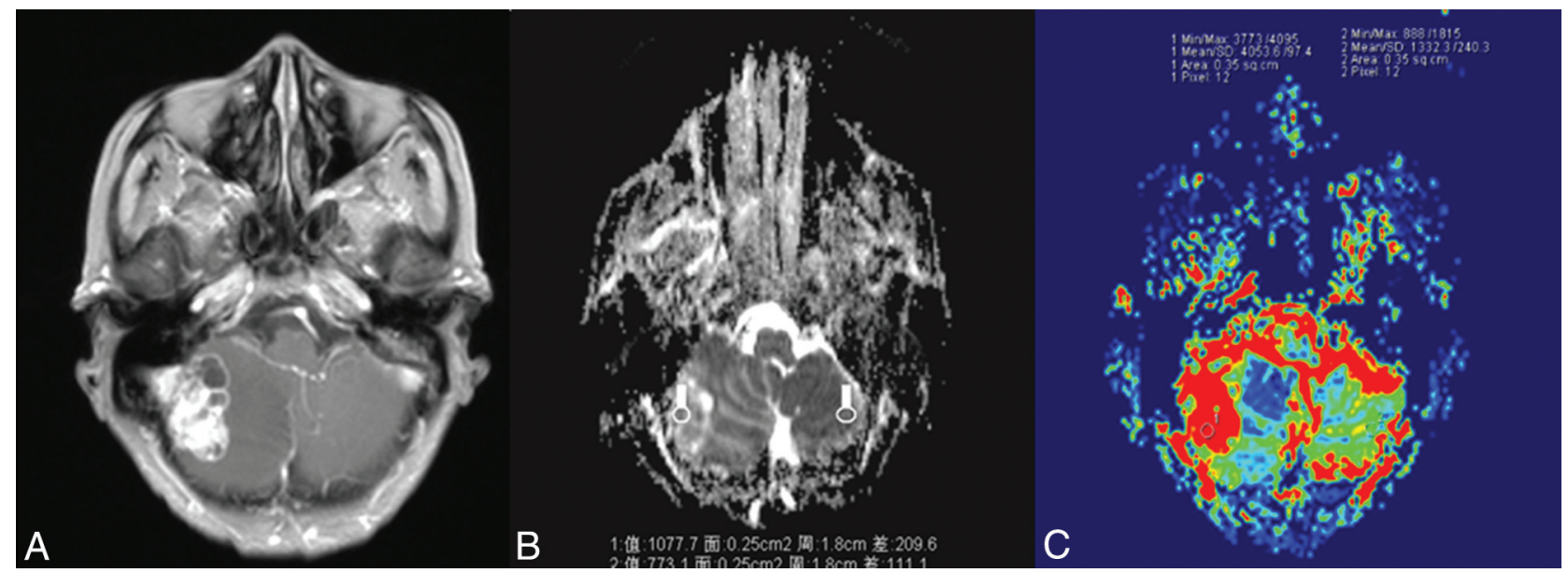

FIG 2. A 70-year-old man with a single brain metastasis. A, Contrast-enhanced axial T1-weighted image demonstrates a contrast-enhancing lesion on the right cerebellar hemisphere. $B$, A corresponding $A D C$ map shows the tumor to have a slightly increased $A D C$ value $(A D C$ min $=$ $\left.1077.7 \times 10^{-3} \mathrm{~mm}^{2} / \mathrm{s}, \mathrm{rADC}=1.39\right)$. $C$, A correlative color $C B V$ image shows moderately elevated perfusion with the calculated $r C B V_{\max }$ of 3.04 .

Table 4: Measures of sensitivity, specificity, PPV, NPV, accuracy, and AUC of ADC min values, rADC ratios, and rCBV $_{\text {max }}$ ratios for differentiation of hemangioblastomas and brain metastases

\begin{tabular}{lccccccc}
\hline & TV & Sensitivity & Specificity & PPV & NPV & Accuracy & AUC \\
\hline $\mathrm{ADC}_{\min }$ & 1.10 & $90.48 \%$ & $96.67 \%$ & $95.0 \%$ & $93.5 \%$ & $94.12 \%$ & 0.968 \\
$\mathrm{rADC}$ & 1.54 & $95.24 \%$ & $96.67 \%$ & $95.2 \%$ & $96.7 \%$ & $96.08 \%$ & 0.971 \\
$\mathrm{rCBV}_{\max }$ & 6.59 & $95.24 \%$ & $53.33 \%$ & $58.8 \%$ & $94.1 \%$ & $70.59 \%$ & 0.756 \\
\hline
\end{tabular}

Note:-PPV indicates positive predictive value; NPV, negative predictive value; TV, threshold value; AUC, area under the curve.

gioblastomas demonstrated significantly higher $\mathrm{ADC}_{\text {min }}$ and $\mathrm{rCBV}_{\text {max }}$ values than those with brain metastases.

Hemangioblastomas typically present in the cerebellar hemisphere and in the third-through-fifth decades of life. Hemangioblastomas have been traditionally described as a marked enhancing mural nodule with a large surrounding cyst. Large draining and feeding vessels within the periphery and solid nodule are commonly seen in hemangioblastomas. Therefore, when MR imaging shows a typically cystic mass with a solid enhancing mural nodule and internal/periphery vessels located in the cerebellum in a younger adult, the most probable diagnosis is hemangioblastoma. These MR imaging features were found in $57.1 \%$ of the hemangioblastomas and in only $20 \%$ of brain metastases in our study. Whereas when a solitary necrotic tumor with heterogeneous enhancement is found in older adults on the MR images, the most probable diagnosis is brain metastasis. However, when an intense enhancing solid tumor without internal/periphery dilated vessels is demonstrated, as in $42.9 \%$ of the hemangioblastomas, there is less certainty as to whether the tumor is a hemangioblastoma or a single brain metastasis. Furthermore, the diagnosis was also confusing when a hemangioblastoma occurred in a 70year-old patient in our series, whose age favors a diagnosis of brain metastases.

DWI has been widely used to evaluate brain tumors, ischemic stroke, abscesses, and other intracranial diseases and has become an indispensable part of brain MR imaging protocols. ADC values represent the mobility of free water molecules within tissue and appear to be correlated with the tumor cellularity, which might be 


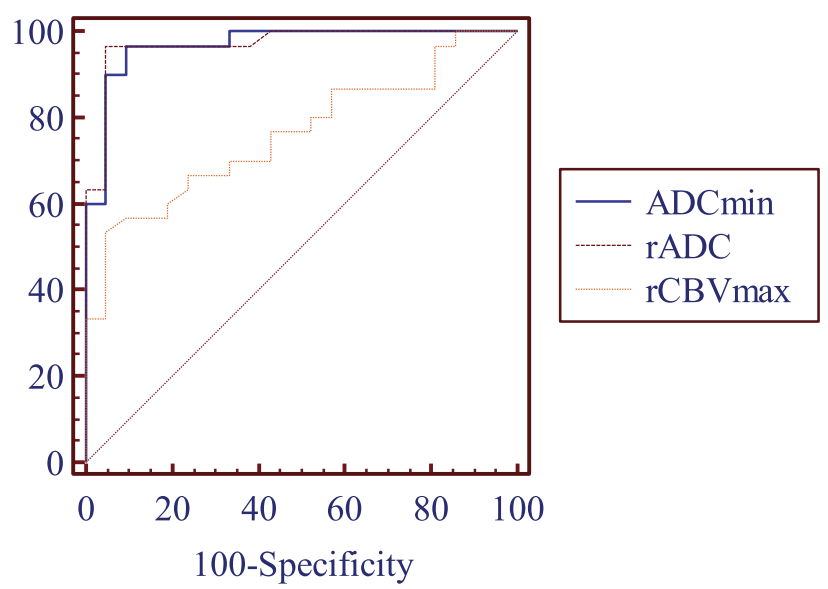

FIG 3. Comparison of ROC curves of $A D C_{\text {min }}, r A D C$, and $r C B V_{\text {max }}$ in the contrast-enhancing lesions for differentiating hemangioblastomas from brain metastases.

a useful adjunct in the preoperative management of patients with common brain tumors, including cerebellar tumors. There are few studies in the literature regarding the DWI findings of hemangioblastomas. In a small group study of 22 cerebellar tumors, Quadery and Okamoto ${ }^{12}$ reported that the ADC values were increased $\left(\geq 1.27 \pm 0.14 \times 10^{-3} \mathrm{~mm}^{2} / \mathrm{s}\right)$ in 10 hemangioblastomas, while other tumors, including 3 metastatic tumors, had lower or similar ADC values compared with those of cerebellar parenchyma (without statistical analysis). ${ }^{20}$ In this study, we found that the signal intensity in the solid contrast-enhancing portions of hemangioblastomas tended to be hypointense or isointense relative to normal-appearing white matter on DWI. In addition, they had significantly lower signal intensity than brain metastases. Furthermore, the ADC values of solid contrast-enhancing areas in this study were higher than those of normal brain parenchyma in all hemangioblastomas, consistent with previous findings. ${ }^{12}$ To the best of our knowledge, the application of ADC values obtained from DWI in distinguishing hemangioblastomas from brain metastases has not been studied previously. This study also revealed that the rate of water diffusion of hemangioblastomas, as reflected by ADC values, was significantly higher than that of brain metastases $(P<.001)$. Several studies have reported that calculated $\mathrm{ADC}$ values were inversely related to tumor cellular attenuation and tumor nucleus/cytoplasm ratios in terms of water diffusivity within intracranial tumors. ${ }^{21,22}$

Densely packed tumor cells can inhibit effective movement of free water molecules and can, therefore, restrict diffusion. Low diffusion could translate into high signal intensity on DWI, with low values on ADC maps. Histopathologically, hemangioblastomas are World Health Organization grade I tumors characterized by stromal cells with plump foamy cytoplasm and an abundant capillary network. ${ }^{20}$ Lower signal intensity on DWI combined with higher ADC values in hemangioblastomas may reflect the lower cellular attenuation and nucleus/cytoplasm ratios compared with metastatic tumors. Furthermore, high vascular spaces in hemangioblastomas may also result from low signal on DWI with increased ADC values. ${ }^{12}$ In a study of 26 metastatic brain lesions, Hayashida et $\mathrm{al}^{23}$ found that the signal intensity of welldifferentiated adenocarcinomas on DWI tended to be signifi- cantly lower than that of poorly differentiated adenocarcinomas and lesions other than adenocarcinomas. They reported that the ADC values of the solid portions of brain metastases were correlated with tumor cellularity and that the signal intensity on DWI may predict the histology of brain metastases. ${ }^{23}$ Therefore, our findings suggest that preoperative determination of the ADC values of the cerebellar tumors in adults may aid in the differential diagnosis of hemangioblastomas and brain metastases.

rCBV measurement derived from DSC-PWI is a useful parameter to evaluate tumor angiogenesis and to differentiate various types of the brain tumors because it adds functional information not available with conventional MR imaging. ${ }^{13-15}$ In vivo measurement of rCBV has been shown to correlate with tumor vascularity and serve as an indicator of tumor grade. ${ }^{14,24}$ In addition, rCBV has also been demonstrated to strongly correlate with vascular endothelial growth factor expression in nonenhancing gliomas. ${ }^{25}$ A maximum rCBV value of $9.4 \pm 2.37$ or $7.7 \pm 1.0$ for hemangioblastomas has been identified in 2 different studies. ${ }^{16,26}$ Hakyemez et al ${ }^{18}$ showed that the rCBV ratios of 6 hemangioblastomas were significantly higher than those of 25 brain metastases $(11.4 \pm 4.40$ versus $5.3 \pm 3.22, P<.05)$. In our group of hemangioblastomas, the $\mathrm{rCBV}_{\text {max }}$ value calculated from the solid contrast-enhancing portion was $8.5 \pm 2.45$, significantly higher than that of brain metastases $(6.4 \pm 2.0)$. The $\mathrm{rCBV}_{\max }$ value for hemangioblastomas or metastases is in good agreement with values reported previously. ${ }^{16,18,26}$

Hemangioblastomas have abundant tumor vessels within tumors due to overexpression of vascular endothelial growth factor, ${ }^{20,27}$ which causes significantly increased rCBV. However, the histopathologic features of brain metastatic tumor vessels have not been established because of a variety of macroscopic and microscopic features connected to the histopathologic characteristics of the primary tumor. Tsougos et $\mathrm{al}^{28}$ demonstrated that the rCBV value of metastases was $10.80 \pm 5.13$ in a study consisting of 6 lung and 8 breast primary tumors. Gaudino et $\mathrm{al}^{29}$ showed that the $\mathrm{rCBV}$ value in lesions was higher $(3.30 \pm 1.59)$ than that of white matter in a series of 59 solitary brain metastases. Our study showed that the rCBV value of brain metastases was $5.3 \pm 3.22$, which is not consistent with that in previous studies, probably due to the heterogeneity of brain metastases of different primary tumors. Thus, brain metastases originating from melanoma or breast carcinoma, which are highly vascular, may present with higher $\mathrm{rCBV}$ than those from lung cancer, known to be less vascular.

In this study, the rCBV values in lesions were found to be markedly high $(>7.5)$ in 2 breast carcinomas and 1 melanoma. More defined rCBV values in brain metastases are worth further investigation with larger sample size. Regardless, our results suggest that high $\mathrm{rCBV}_{\max }$ appears to be indicative of hemangioblastomas, whereas a low $\mathrm{rCBV}_{\max }$ is suggestive of brain metastases. One could assume that when an intense-enhancing solid tumor in the posterior fossa presents with a marked increase of $\mathrm{rCBV}$, the most probable diagnosis is hemangioblastoma. In this case, preoperative embolization should be considered to control inaccessible arterial supply and reduce the tumor vascularity, which could aid in the resection of the hemangioblastoma. This approach is often used in spinal hemangioblastoma to reduce the 
operative complication rates and intraoperative bleeding. ${ }^{9,30,31}$ Therefore, DSC-PWI may be helpful for both accurate preoperative diagnosis of hemangioblastomas and postembolization follow-up.

All 3 MR imaging parameters were significant for contrastenhancing tumoral regions to differentiate hemangioblastomas from brain metastases. However, from the ROC analysis, the sensitivity, specificity, and accuracy levels were significantly higher for diffusion parameters (accuracy for rADC, 96.08\%) than for perfusion parameters (accuracy for $\mathrm{rCBV}_{\max }, 70.59 \%$ ). In addition, there was moderate overlap of the $\mathrm{rCBV}_{\text {max }}$ parameter for differentiating hemangioblastomas and brain metastases, with a specificity of only $53.33 \%$. Thus, this study indicated that rADC is a robust parameter with high sensitivity $(95.24 \%)$ and specificity (96.67\%), which may be helpful in differentiating hemangioblastomas from brain metastases. As a part of a multiparametric MR imaging protocol, MR spectroscopy may allow further characterization of intracranial tumor by providing metabolic information about the tumor tissue. ${ }^{32,33}$ It has been widely reported that Cho levels correlate with the degree of malignancy in brain tumor and are linearly correlated with cell density. ${ }^{33}$ To our knowledge, no study has evaluated the ability of MR spectroscopy in the differentiation of hemangioblastomas from brain metastases.

There are some limitations to our study. The most significant one was its retrospective nature, which may have led to bias in case selection. Another potential limitation was that the number of the metastatic tumors originating from primary sites other than lung were few. Further prospective studies with a larger number of metastatic tumors are required. Third, we could not exclude the presence of tiny intratumoral hemorrhage within lesions that may result in susceptibility blooming, and in turn interfere with DWI and PWI evaluation, though there was no obvious evidence of hemorrhage on conventional MR imaging and DWI. It is also recognized that the lack of a susceptibility sequence in the MR imaging protocol was also a limitation because that would have been helpful in excluding hemorrhagic metastasis.

\section{CONCLUSIONS}

This study demonstrates that DWI and DSC-PWI MR imaging measurements in the contrast-enhancing tumoral region allow differentiation of hemangioblastomas from brain metastases. On DWI, higher $\mathrm{rADC}$ and $\mathrm{ADC}_{\text {min }}$ values in hemangioblastomas than in the brain metastases were the most consistent finding in our study. Therefore, DWI appears to be an efficient MR imaging technique for the possible differentiation of hemangioblastomas from brain metastases.

\section{REFERENCES}

1. Louis DN, Ohgaki H, Wiestler OD, et al. The 2007 WHO classification of tumours of the central nervous system. Acta Neuropathol 2007;114:97-109 CrossRef Medline

2. Young S, Richardson AE. Solid haemangioblastomas of the posterior fossa: radiological features and results of surgery. J Neurol $\mathrm{Neu}$ rosurg Psychiatry 1987;50:155-58 CrossRef Medline

3. Yoshida S, Takahashi H. Cerebellar metastases in patients with cancer. Surg Neurol 2009;71:184-87; discussion 187 CrossRef Medline

4. Kanner AA, Suh JH, Siomin VE, et al. Posterior fossa metastases: aggressive treatment improves survival. Stereotact Funct Neurosurg 2003;81:18-23 CrossRef Medline
5. Alexandru D, Bota DA, Linskey ME. Epidemiology of central nervous system metastases. Prog Neurol Surg 2012;25:13-29 CrossRef Medline

6. Bründl E, Schödel P, Ullrich OW, et al. Surgical resection of sporadic and hereditary hemangioblastoma: our 10-year experience and a literature review. Surg Neurol Int 2014;5:138 CrossRef Medline

7. Tabei Y, Miyamoto S, Suzuki I. Multidisciplinary approach to management of patients with brain metastases [in Japanese]. Gan To Kagaku Ryoho 2013;40:1288-94 Medline

8. Le Reste PJ, Henaux PL, Morandi X, et al. Sporadic intracranial haemangioblastomas: surgical outcome in a single institution series. Acta Neurochir (Wien) 2013;155:1003-09; discussion 1009 CrossRef Medline

9. Sakamoto N, Ishikawa E, Nakai Y, et al. Preoperative endovascular embolization for hemangioblastoma in the posterior fossa. Neurol Med Chir (Tokyo) 2012;52:878-84 CrossRef Medline

10. Schaefer PW, Grant PE, Gonzalez RG. Diffusion-weighted MR imaging of the brain. Radiology 2000;217:331-45 CrossRef Medline

11. Kang Y, Choi SH, Kim YJ, et al. Gliomas: histogram analysis of apparent diffusion coefficient maps with standard- or high-b-value diffusion-weighted MR imaging-correlation with tumor grade. Radiology 2011;261:882-90 CrossRef Medline

12. Quadery FA, Okamoto K. Diffusion-weighted MRI of haemangioblastomas and other cerebellar tumours. Neuroradiology 2003;45: 212-19 Medline

13. Kickingereder P, Wiestler B, Sahm F, et al. Primary central nervous system lymphoma and atypical glioblastoma: multiparametric differentiation by using diffusion-, perfusion-, and susceptibilityweighted MR imaging. Radiology 2014;272:843-50 CrossRef Medline

14. Guzmán-De-Villoria JA, Mateos-Pérez JM, Fernández-García P, et al. Added value of advanced over conventional magnetic resonance imaging in grading gliomas and other primary brain tumors. Cancer Imaging 2014;14:35 CrossRef Medline

15. Ho CY, Cardinal JS, Kamer AP, et al. Relative cerebral blood volume from dynamic susceptibility contrast perfusion in the grading of pediatric primary brain tumors. Neuroradiology 2015;57:299-306 CrossRef Medline

16. Kumar VA, Knopp EA, Zagzag D. Magnetic resonance dynamic susceptibility-weighted contrast-enhanced perfusion imaging in the diagnosis of posterior fossa hemangioblastomas and pilocytic astrocytomas: initial results. J Comput Assist Tomogr 2010;34:825-29 CrossRef Medline

17. She DJ, Xing Z, Zeng Z, et al. Differentiation of hemangioblastomas from pilocytic astrocytomas using 3-T magnetic resonance perfusion-weighted imaging and MR spectroscopy. Neuroradiology 2015; 57:275-81 CrossRef Medline

18. Hakyemez B, Erdogan C, Bolca N, et al. Evaluation of different cerebral mass lesions by perfusion-weighted MR imaging. J Magn Reson Imaging 2006;24:817-24 CrossRef Medline

19. Wetzel SG, Cha S, Johnson G, et al. Relative cerebral blood volume measurements in intracranial mass lesions: interobserver and intraobserver reproducibility study. Radiology 2002;224:797-803 CrossRef Medline

20. Cuccurullo L, Prudente ME, Maffia S, et al. An ultrastructural study of the histogenesis of haemangioblastoma. Pathologica 2009;101: 1-5 Medline

21. Guo AC, Cummings TJ, Dash RC, et al. Lymphomas and high-grade astrocytomas: comparison of water diffusibility and histologic characteristics. Radiology 2002;224:177-83 CrossRef Medline

22. Barajas RF Jr, Rubenstein JL, Chang JS, et al. Diffusion-weighted MR imaging derived apparent diffusion coefficient is predictive of clinical outcome in primary central nervous system lymphoma. AJNR Am J Neuroradiol 2010;31:60-66 CrossRef Medline

23. Hayashida Y, Hirai T, Morishita S, et al. Diffusion-weighted imaging of metastatic brain tumors: comparison with histologic type and tumor cellularity. AJNR Am J Neuroradiol 2006;27:1419-25 Medline

24. Jain R, Gutierrez J, Narang J, et al. In vivo correlation of tumor blood volume and permeability with histologic and molecular angiogenic 
markers in gliomas. AJNR Am J Neuroradiol 2011;32:388-94 CrossRef Medline

25. Maia AC Jr, Malheiros SM, da Rocha AJ, et al. MR cerebral blood volume maps correlated with vascular endothelial growth factor expression and tumor grade in nonenhancing gliomas. AJNR Am J Neuroradiol 2005;26:777-83 Medline

26. Bing F, Kremer S, Lamalle L, et al. Value of perfusion MRI in the study of pilocytic astrocytoma and hemangioblastoma: preliminary findings [in French]. J Neuroradiol 2009;36:82-87 CrossRef Medline

27. Hussein MR. Central nervous system capillary haemangioblastoma: the pathologist's viewpoint. Int J Exp Pathol 2007;88: 311-24 CrossRef Medline

28. Tsougos I, Svolos P, Kousi E, et al. Differentiation of glioblastoma multiforme from metastatic brain tumor using proton magnetic resonance spectroscopy, diffusion and perfusion metrics at $3 \mathrm{~T}$. Cancer Imaging 2012;12:423-36 CrossRef Medline
29. Gaudino S, Di Lella GM, Russo R, et al. Magnetic resonance imaging of solitary brain metastases: main findings of nonmorphological sequences. Radiol Med 2012;117:1225-41 CrossRef Medline

30. Biondi A, Ricciardi GK, Faillot T, et al. Hemangioblastomas of the lower spinal region: report of four cases with preoperative embolization and review of the literature. AJNR Am J Neuroradiol 2005;26: 936-45 Medline

31. Chu BC, Terae S, Hida K, et al. MR findings in spinal hemangioblastoma: correlation with symptoms and with angiographic and surgical findings. AJNR Am J Neuroradiol 2001;22: 206-17 Medline

32. Horská A, Barker PB. Imaging of brain tumors: MR spectroscopy and metabolic imaging. Neuroimaging Clin N Am 2010;20:293-310 CrossRef Medline

33. Brandão LA, Castillo M. Adult brain tumors: clinical applications of magnetic resonance spectroscopy. Neuroimaging Clin N Am 2013; 23:527-55 CrossRef Medline 